\title{
Quantification of $\mathrm{PEG}_{40}$ St squeeze out from DSPC/PEG ${ }_{40}$ St monolayers at higher molar ratios
}

Sevgi Kilic

Department of Chemical Engineering, Izmir Institute of Technology, Urla, Izmir, 35430, Turkey

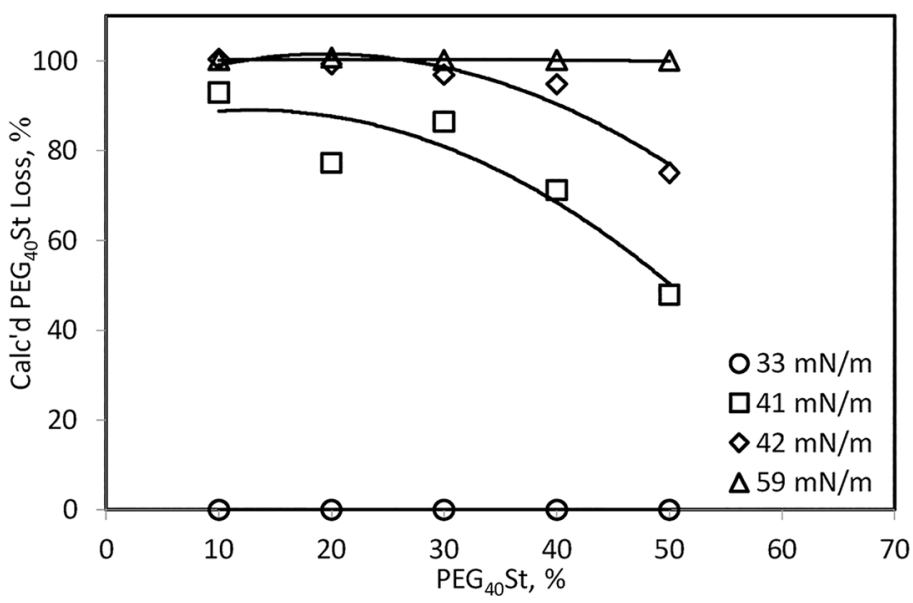

A R T I C L E I N F O

\section{Keywords:}

Lipid

Emulsifier

DSPC

$\mathrm{PEG}_{40} \mathrm{St}$

Squeeze out

Langmuir trough

Microbubble

Ultrasound contrast agent

\begin{abstract}
A B S T R A C T
Mixtures of 1,2 Distearoyl-sn-glycero-3-phosphocholine (DSPC) and polyoxyethylene-40-stearate (PEG $40 \mathrm{St}$ ) were prepared at different molar ratios and their miscibility were investigated using Langmuir isotherms. Pure DSPC monolayer exhibited a liquid-condensed (LC) phase whereas PEG $_{40}$ St monolayer exhibited liquid-expanded (LE) phase at the air-water interface at $22 \pm 2{ }^{\circ} \mathrm{C}$. At the collapse pressure of $33 \mathrm{mN} / \mathrm{m}$, the PEG $40 \mathrm{St}$ mean molecular area was calculated to be $28 \AA^{2} /$ molecule for 9:1 composition and $50 \AA^{2} /$ molecule for $5: 5$ composition, showing an increasing trend with the emulsifier content. A quantification method was developed to estimate the squeeze out amount of $\mathrm{PEG}_{40}$ St from Langmuir isotherms of the DSPC/PEG ${ }_{40}$ St mixtures at different molar ratios. Almost 93\%, 82\%, and 53\% of $\mathrm{PEG}_{40}$ St displaced for the 9:1, 7:3, and 5:5 mixtures, respectively, at the end of the first collapse plateau and showed a decreasing trend with the $\mathrm{PEG}_{40}$ St content. Remaining $\mathrm{PEG}_{40}$ St squeezed out at the end of the second collapse plateau, where $20 \%$ of $\mathrm{PEG}_{40} \mathrm{St}$ still contained within the 5:5 composition. It was concluded that increasing $\mathrm{PEG}_{40}$ St content would be advantageous to design more stable lipid based microbubbles.
\end{abstract}

E-mail address: sevgikilic@iyte.edu.tr. 


\section{Introduction}

Lipid-based microbubbles are micron size gas bubbles used in medicine as ultrasound contrast agent to improve the quality of ultrasound image [1] as well as efficient drug and gene delivery. [1-4] However, phospholipids alone are not capable of forming microbubbles [5,6], and an additional component, emulsifier, is needed [7-10]. The lipid used in a microbubble shell is usually a phosphocholine, DSPC, and the emulsifier could be Tween-40, DSPE-PEG ${ }_{5000}$, DSPE-PEG 2000 , or $\mathrm{PEG}_{40} \mathrm{St}$, among which $\mathrm{PEG}_{40} \mathrm{St}$ is particularly attracted in the lipid microbubbles [11-17]. The PEG chains also protect the gas bubble from aggregation in the bloodstream $[18,19]$. In a microbubble formulation, DSPC:PEG ${ }_{40}$ St mixture with a molar ratio of 9:1 has usually been used [13,15,20-25]. To the best of our knowledge, the miscibility behavior of DSPC:PEG ${ }_{40}$ St at molar ratio higher than $15 \mathrm{~mol}_{0}$ of $\mathrm{PEG}_{40} \mathrm{St}$ has not been studied in the literature [6,26,27].

The miscibility characteristics of DSPC/ $\mathrm{PEG}_{40} \mathrm{St}$ mixtures can be estimated by a relatively simple Langmuir monolayer technique where surface pressure of a monolayer is measured with a Wilhelmy plate while compressing a flat 2D-monolayer at the air-water interface $[28,29]$. The measured average area per molecule, $A_{12}$, can be obtained experimentally by dividing the trough area to the number of molecules in the mixture [6]. The measured mean molecular area is the cumulative area occupied by each component, $\left.\Sigma x_{i}<A_{i}\right\rangle$, in the monolayer, $A_{12}=x_{1} .\left\langle A_{1}\right\rangle+x_{2} \cdot\left\langle A_{2}\right\rangle$. However, the true mean molecular area could not be accurately estimated for each component during the measurements due to attractive-repulsive forces between the component molecules and their complexations [30-33]. The mixture could be considered "ideal" if the component molecules have no irregular packing attachments, no complex formations, and form a phase separation in the monolayer $[6,30]$. In such cases, the ideal average mean molecular area, $A_{12}{ }^{\text {ideal }}$, can be related to the area per molecule, $A_{i}$, for each component obtained from their pure component isotherms at the specified surface pressure and mole fraction of each species, $A_{12}{ }^{\text {ideal }}=x_{1} \cdot A_{1}+x_{2} \cdot A_{2}[6,30]$. Although the surface pressure-area isotherms enable to investigate the physical interactions of components on the monolayer, deviations from the ideality is possible such that the surface area of the trough is large and bounded by the hydrophobic barriers, the compression is anisotropic and unidirectional, and the surface pressure may not be measured accurately during the transition from a fluid to solid phase [29]. However, these artifacts are small and reliable data can be extracted from the Langmuir mean molecular areasurface pressure isotherms for the monolayers [28].

Different regions of the Langmuir isotherm can give information on the phase behavior of the molecules for each component. It was shown that more ordered and rigid monolayers collapse at relatively higher surface pressures, usually by fracturing, followed by loss of materials to the air side with the formation of multilayered aggregates, or to the sub phase with budding or vesicle formation [25,29,31,34]. When a binary mixture of components having two different collapse pressures were compressed, at least two plateaus will exhibit, one is at the lower collapse pressure for the expanded phase and the other is at the higher collapse pressure for the more rigid component [6,35-38]. The component in the expanded phase usually squeeze out from the monolayer over the plateau region $[6,34]$.

$\mathrm{PEG}_{40} \mathrm{St}$ was shown to squeeze out from the monolayer at higher compression pressures and, to our knowledge, no studies were found to quantify the amount of $\mathrm{PEG}_{40} \mathrm{St}$ departed from the monolayer during the collapse plateaus. In this study, we aimed to investigate the DSPC/ $\mathrm{PEG}_{40}$ St mixtures at higher molar ratios from 9:1 to 5:5 compositions. To the best of our knowledge, the miscibility of DSPC:PEG ${ }_{40} \mathrm{St}$ at molar ratios higher than $15 \mathrm{~mol} \%$ of $\mathrm{PEG}_{40} \mathrm{St}$ has not been studied in the literature $[6,26,27]$. The surface pressure versus area isotherms were measured on a Langmuir trough for the mixtures and analyzed for their compression, collapse, molecular area, and squeeze-out properties in a two dimensional (2D) monolayer technique. It was found that $\mathrm{PEG}_{40} \mathrm{St}$ in $10 \%$ composition is easily squeeze-out from the $2 \mathrm{D}$ film up to about $93 \%$ at the end of the first collapse plateau, at $41 \mathrm{mN} / \mathrm{m}$, whereas almost $60 \% \mathrm{PEG}_{40}$ St squeezed out from the monolayer for the 5:5 composition, retaining almost $40 \%$ of $\mathrm{PEG}_{40} \mathrm{St}$ molecules in the $2 \mathrm{D}$ film. Using $\mathrm{PEG}_{40} \mathrm{St}$ at higher concentrations was found advantageous not only to increase the stability of the microbubbles but also reduce the product cost for the ultrasound contrast agent.

\section{Materials and methods}

\subsection{Materials}

1,2 Distearoyl-sn-glycero-3-phosphocholine (DSPC, 99\%) and Polyoxyethylene-40- stearate $\left(\mathrm{PEG}_{40} \mathrm{St}\right)$ were purchased from Sigma Aldrich (St. Louis, MO). Chloroform $\left(\mathrm{CHCl}_{3}, 99-99.4 \%\right)$ was purchased from Merck and used as a solvent to prepare spreading solutions. Ultrapure water used as subphase was produced by Millipore purification system with specific resistivity of $18 \mathrm{M} \Omega . \mathrm{cm}$. Predetermined amount of components were weighted into a clean vial and dissolved in chloroform resulting a concentration less than $1.0 \mathrm{mg} / \mathrm{ml}$. DSPC/ $\mathrm{PEG}_{40}$ St mixtures were prepared at molar ratios of 9:1; 8:2; 7:3; 6:4 and $5: 5$. After chloroform addition, the vials were immediately sealed with screw cap to avoid chloroform evaporation and, if needed, stored in the freezer at $-22^{\circ} \mathrm{C}$. Before spreading the solution at the gas-liquid interface, the sealed vial was homogenized using a bath sonicator and continuously stirred by hand-shaking with the cap closed.

\subsection{Langmuir isotherm measurements}

Langmuir-Blodgett system (KSV minitrough, Finland) with two movable PTFE barriers was used to study the phase behavior of the binary systems and pure components. The surface area of the trough is $273 \mathrm{~cm}^{2}$ with dimensions of $364 \times 75 \times 7 \mathrm{~mm}^{3}$ and a subphase volume of $140 \mathrm{ml}$. The system was enclosed in a box to minimize possible contamination of air-monolayer-water interface and the disturbance of the monolayer by air currents. Trough was filled with ultrapure water with specific resistivity of $18 \mathrm{M} \Omega \mathrm{cm}$ produced by a Millipore purification system. Cleanness of the air-water interface was confirmed by closing and opening the barriers and ensuring that surface pressure readings do not differ by more than $\pm 0.1 \mathrm{mN} / \mathrm{m}$. The spreading solutions were spread on the water subphase via Hamilton micro syringe. The monolayer was allowed to evaporate the chloroform for $20 \mathrm{~min}$. The surface pressure-area $(\pi-\mathrm{A})$ isotherms were obtained via symmetric compression of monolayers by the two barriers. A compression speed of $5 \mathrm{~mm} / \mathrm{min}$ was used in all experiments. Each isotherm was performed 4-5 times to ensure reproducibility of the isotherms.

\section{Results and discussion}

DSPC and $\mathrm{PEG}_{40}$ St mixtures at different molar ratios were prepared and their miscibility behaviors were investigated using Langmuir isotherms. The Langmuir isotherms are shown in Fig. 1a where the surface pressure $(\pi)$ versus mean area per molecule isotherms were measured on a Langmuir trough for pure DSPC, pure $\mathrm{PEG}_{40} \mathrm{St}$, and their binary mixtures at DSPC/PEG ${ }_{40}$ St molar ratios of 9:1, 8:2, 7:3, 6,:4, and 5:5. As shown in the figure, the isotherms show similar shape and behavior for all the mixtures, therefore, for simplicity, main discontinuities as the turning points were marked in Fig. 1b by the arrows on the isotherms for the pure components and on the isotherm for their 8:2 mixture. As shown in the figure, pure DSPC monolayer exhibited a liquid-condensed (LC) phase at the air-water interface upon spreading at room temperature. A steep increase in the surface pressure was evident for the DSPC with little compression occurred with its mean molecular area. The steep surface pressure change may indicate that DSPC form a continuous ordered monolayer at the interface with a rigid condensed phase. The surface pressure of DSPC at about $59 \mathrm{mN} / \mathrm{m}$ almost 

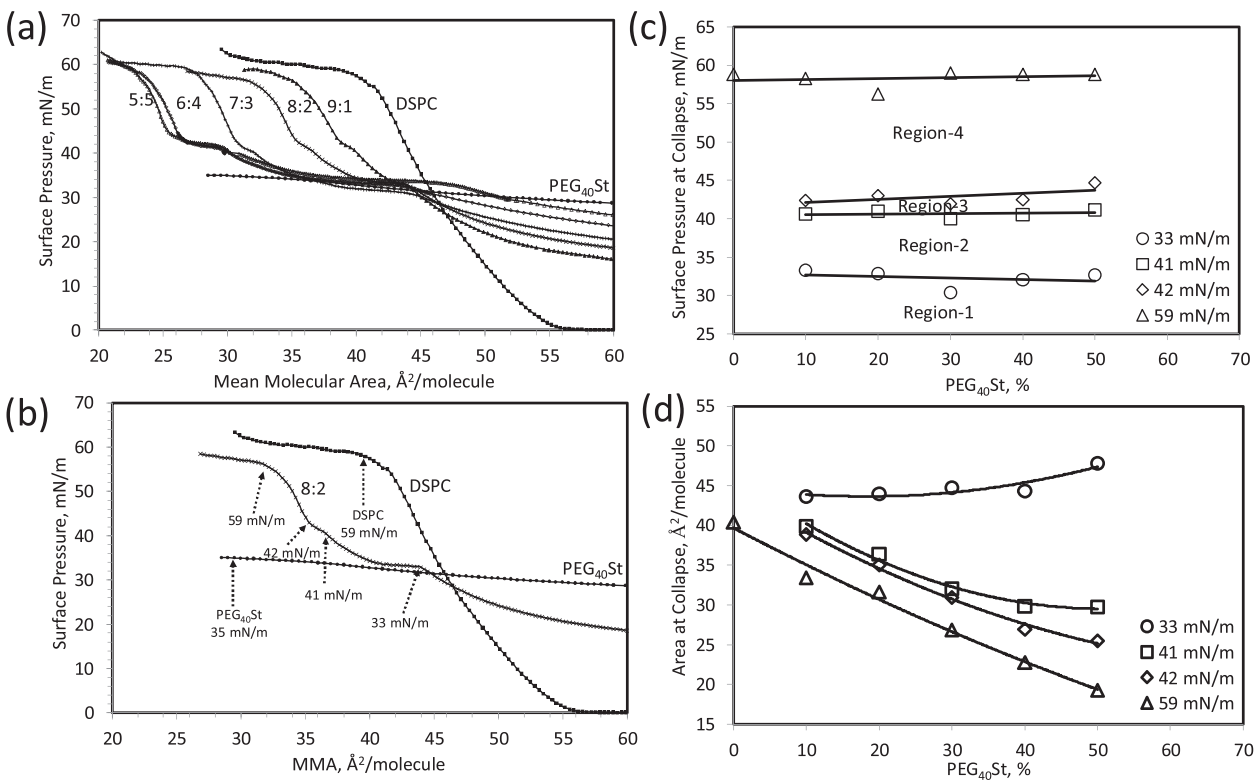

Fig. 1. (a) The surface pressure-mean molecular area $(\pi-\mathrm{A})$ isotherms of pure DSPC, pure $\mathrm{PEG}_{40} \mathrm{St}$, and isotherms for DSPC/PEG ${ }_{40} \mathrm{St}$ mixtures with different compassions at the air/ water interface, (b) main turning points marked on the isotherm for the 8:2 mixture, (c) the collapse pressures for each mixture and the plateau regions on the isotherms, and (d) the mean molecular areas at the collapse pressures for the mixtures. unchanged as the area of the trough decreased significantly indicating that the DSPC molecules departed from the two dimensional monolayer forming a double layer on the existing 2D monolayer called a collapse. Therefore, the collapse pressure for DSPC was measured to be about $59 \mathrm{mN} / \mathrm{m}$ which is in good agreement with the literature [30,39,40]. At the collapse pressure, the mean molecular area for DSPC was found to be about $40 \AA^{2}$ /molecule, which is also in good agreement with the literature $[30,39,40]$. It was understood that the DSPC monolayer in the liquid-condensed phase showed strong cohesive interactions between $\mathrm{C}_{18}$ aliphatic chains on aqueous subphase and the surface pressures increased significantly with little increase in the mean molecular area, making the DSPC molecules rigid in nature [41-43].

Unlike DSPC, $\mathrm{PEG}_{40} \mathrm{St}$ monolayer exhibited a non-zero surface pressure even at very low molecular densities. The higher surface pressure suggests the presence of intermolecular interactions in $\mathrm{PEG}_{40} \mathrm{St}$ chains forming globular aggregates on the surface of the aqueous subphase. The surface pressure for the pure $\mathrm{PEG}_{40} \mathrm{St}$ increased only little as large changes occurred in its mean molecular area. The negligible surface pressure change indicated that the emulsifier molecules did not form a continuous ordered monolayer at the air-water interface with a rigid condensed phase, but spread as floating clusters due to intermingling of polymer chains bound at the interface in a pancake conformation [44]. As evidenced also from the compressibility $\left(\mathrm{C}_{\mathrm{s}}{ }^{-1}\right)$ curve for the $\mathrm{PEG}_{40} \mathrm{St}$ (data not shown), the Langmuir isotherm of $\mathrm{PEG}_{40}$ St exhibited a collapse pressure at about $35 \mathrm{mN} / \mathrm{m}$, consistent with the literature $[39,45]$. There are inconsistencies in the literature about the mean molecular area of $\mathrm{PEG}_{40} \mathrm{St}$ at its collapse pressure such that the mean molecular areas of $40 \AA^{2} /$ molecule [46], $30 \AA^{2} /$ molecule [27], and $20 \AA^{2} /$ molecule [11] were reported. Here, our measurements showed that the mean molecular area for $\mathrm{PEG}_{40} \mathrm{St}$ is about $30 \AA^{2} / \mathrm{mo}$ lecule at the collapse pressure of $35 \mathrm{mN} / \mathrm{m}$.

Langmuir isotherms of the DSPC/ $\mathrm{PEG}_{40}$ St mixtures were located in between the isotherms for pure DSPC and pure $\mathrm{PEG}_{40} \mathrm{St}$. There are four discontinuities as the turning points and four plateau regions in the mixture isotherms as illustrated in $b$ on the isotherm for the $8: 2$ mixture as an example. The values for the surface pressures and the mean molecular areas for the isotherms at different $\mathrm{PEG}_{40} \mathrm{St}$ content are also shown in Fig. 1c and d respectively. The first plateau region, region-1, is below the first collapse pressure of about $33 \mathrm{mN} / \mathrm{m}$ for the mixtures studied and it was lower than $35 \mathrm{mN} / \mathrm{m}$ for the collapse pressure of the pure $\mathrm{PEG}_{40} \mathrm{St}$. The mean molecular areas at the start of the first collapse, $33 \mathrm{mN} / \mathrm{m}$, were obtained to be about $45 \AA^{2} /$ molecule for the mixtures and it showed a slightly increasing trend with the $\mathrm{PEG}_{40} \mathrm{St}$ content. The second plateau region, region-2, is the region started at $33 \mathrm{mN} / \mathrm{m}$ and ended at $41 \mathrm{mN} / \mathrm{m}$. The second collapse initiated at the surface pressures of about $41 \mathrm{mN} / \mathrm{m}$ for the mixtures. Both molecular configurations/reconformations and consequently $\mathrm{PEG}_{40} \mathrm{St}$ squeeze out took place from the monolayer at the air-water interface through this plateau region. Therefore, the mean molecular area decreased with increasing $\mathrm{PEG}_{40} \mathrm{St}$ content of the mixtures at $41 \mathrm{mN} / \mathrm{m}$ as shown in Fig. 1d. The third plateau region, region-3, is the region starts at $41 \mathrm{mN} / \mathrm{m}$ and ends at $42 \mathrm{mN} / \mathrm{m}$ and showed slightly increasing trend. A second collapse was seen in this region therefore a significant decrease in the mean molecular area were obtained for the mixtures with increasing $\mathrm{PEG}_{40} \mathrm{St}$ content. The forth plateau region, region-4, is the region where the shape of the isotherm show somewhat similar trend with the isotherm for the pure DSPC. The surface pressures were obtained to be about $59 \mathrm{mN} / \mathrm{m}$ for the studied DSPC/PEG 40 St mixtures as it was the same with the pure DSPC isotherm. However, the mean molecular area decreased almost linearly with increasing $\mathrm{PEG}_{40} \mathrm{St}$ content indicating that a significant amount of $\mathrm{PEG}_{40} \mathrm{St}$, if not all, squeezed out from the monolayer at the air-water interface.

In the high compressibility region, molecules spread in a more relaxed form and therefore the mixture isotherms exhibited more expanded behavior in the pancake conformation with increasing $\mathrm{PEG}_{40} \mathrm{St}$ content $[32,47]$. The DSPC/ $\mathrm{PEG}_{40} \mathrm{St}$ mixture isotherms exhibited a plateau around the collapse pressure of $\mathrm{PEG}_{40} \mathrm{St}$ and shifted to the left of the DSPC isotherm with increasing emulsifier content. It was reported that the location of the DSPC/PEG ${ }_{40}$ St mixture isotherms up to $15 \%$ of $\mathrm{PEG}_{40}$ St content was independent of the emulsifier content after the plateau region, exhibiting an isotherm identical to that of pure DSPC [6]. Similar behavior was observed with DSPC/DSPE-PEG ${ }_{2000}$, DPPC/PEG 8 St, and Span60/PEG 40 St mixtures $[27,43,48,49]$. Our mixture isotherms studied up to $50 \% \mathrm{PEG}_{40} \mathrm{St}$ contents exhibited plateaus broadening with increasing $\mathrm{PEG}_{40} \mathrm{St}$ content in the monolayer. Enlargement of the plateau was also observed for DSPC/PEG ${ }_{40}$ St mixture elsewhere up to $15 \%$ of $\mathrm{PEG}_{40} \mathrm{St}$ content [6], but isotherms of the Span60/PEG ${ }_{40}$ St mixed monolayers were seen to be intersecting each other at one point [27]. With further compression of the monolayers, additional smaller plateaus were also observed around $42 \mathrm{mN} / \mathrm{m}$. Similar to the first plateau, mixtures exhibited more prominent plateaus with increasing $\mathrm{PEG}_{40} \mathrm{St}$ content. The increase in the extent of second plateau was also seen in Span60/PEG ${ }_{40}$ St mixtures [27]. The second plateaus were attributed to partial removal of the emulsifier from the mixed monolayers [27]. Scaling of the plateaus with the emulsifier 


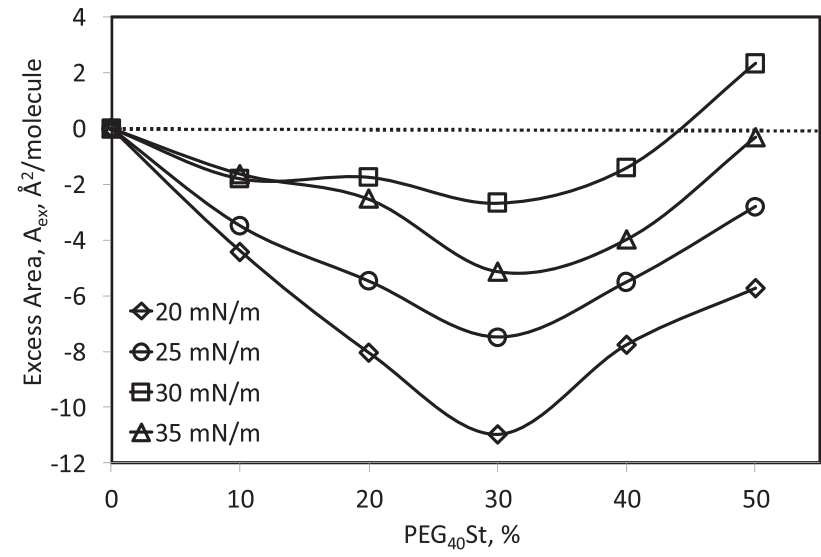

Fig. 2. The excess area of mixing $\left(A_{e x}\right)$ calculated for $D S P C / P G_{40}$ St mixtures at different surface pressures.

content clearly indicated the presence of more emulsifier in the mixed monolayer and thus their progressive elimination from the monolayers.

The surface pressure-area $(\pi-\mathrm{A})$ isotherms provide information on the molecular organization and molecular interaction of the components in mixed monolayers. The excess area $\left(A_{e x}\right)$ can be calculated from the measured mean molecular area $\left(A_{12}\right)$ of the real mixed monolayer at the given surface pressure minus the mean molecular area of the ideal mixture $\left(A_{12}{ }^{\text {ideal }}\right)\left(\mathrm{A}_{\mathrm{ex}}=\mathrm{A}_{12}-\left(\mathrm{x}_{1} \mathrm{~A}_{1}+\mathrm{x}_{2} \mathrm{~A}_{2}\right)\right)[30,50]$, where $x_{1}$ and $x_{2}$ are mole fractions of the components, $A_{1}$ and $A_{2}$ are molecular areas of the pure components at that surface pressure. The zero value for $A_{e x}$ indicates that the components form an ideal mixed monolayer or they are immiscible $[30,43]$. On the other hand, deviation from ideality signify that the components are miscible and form non-ideal mixed monolayers $[30,43]$. To gain more insight on the interaction type of the components and their miscibility behavior, excess areas, $A_{e x}$, of the mixed monolayers were calculated as shown in Fig. 2. As shown in the figure, all mixtures exhibited negative deviations from ideality, highest for the 7:3 composition. These negative deviations were explained by extension of PEG chains into the subphase by conformational changes with compression such that mean molecular area approaches up to the mean molecular area for the stearate chains [43]. Considering the electrostatic repulsions between the polar head groups of the zwitterionic phosphatidylcholines [51,52], shielding effect of neutral $\mathrm{PEG}_{40} \mathrm{St}$ on these electrostatic repulsions may also play an additional role for creation of a more condensed phase. The negative $A_{e x}$ values also show that the tilted and more relaxed pure DSPC molecules may be transformed into a more ordered configuration in the mixed monolayer as well as when the pure $\mathrm{PEG}_{40}$ St molecules in pancake configuration compressed to a more aligned mushroom or brush configuration in the mixed monolayer [35]. Therefore, the measured mean molecular area is smaller than the mean molecular areas measured in their pure ideal states. At higher compressions when the surface pressure increased up to the first collapse pressure of $33 \mathrm{mN} / \mathrm{m}$, the negative excess area calculated for the mixtures decreased significantly and approached to "zero". The zero excess area indicated that the DSPC/PEG 40 St monolayer formed phase separated domains and the measured area could be treated as the ideal area at the first collapse pressure, $A_{12}=A_{12}{ }^{\text {ideal }}=$ $x_{1} \cdot A_{1}+x_{2} \cdot A_{2}$. When the surface pressure exceeds the first collapse pressure of $33 \mathrm{mN} / \mathrm{m}$, the calculated excess area became more negative again at the surface pressure of $35 \mathrm{mN} / \mathrm{m}$ which is above the collapse pressure of $33 \mathrm{mN} / \mathrm{m}$ for the mixture. We believe that there were not only the conformational changes but also a squeeze out of $\mathrm{PEG}_{40} \mathrm{St}$ molecules from the monolayers above the collapse pressure of the mixture, which would mislead any calculation on neither excess area nor other thermodynamic parameters due to mass loss from the monolayer at the air-water interface.

The mean molecular areas were compared for the DSPC/PEG $40 \mathrm{St}$

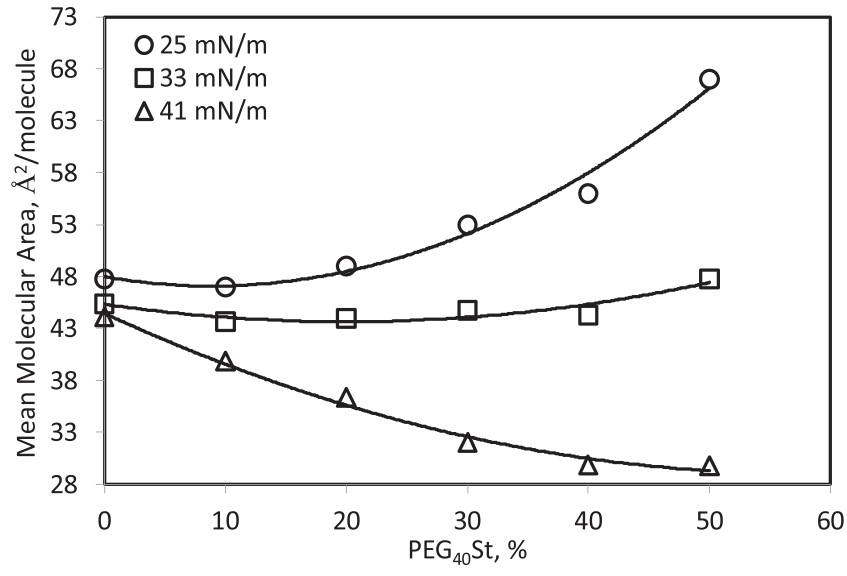

Fig. 3. Mean molecular area of $\mathrm{DSPC} / \mathrm{PEG}_{40} \mathrm{St}$ mixtures below collapse pressure, at the first collapse pressure, $33 \mathrm{mN} / \mathrm{m}$, and at the second collapse pressure, $41 \mathrm{mN} / \mathrm{m}$.

mixtures for different plateau regions as shown in Fig. 3. $\mathrm{PEG}_{40} \mathrm{St}$ molecules are in expanded conformation and occupies larger area at the air-water interface at lower pressures. Therefore, as shown in the figure, the mean molecular areas were measured relatively larger for the $\mathrm{DSPC} / \mathrm{PEG}_{40} \mathrm{St}$ mixtures at pressures lower than the collapse pressure of the mixture. At the first collapse pressure of $33 \mathrm{mN} / \mathrm{m}$, both DSPC and $\mathrm{PEG}_{40} \mathrm{St}$ molecules were significantly compressed to their minimal mean molecular areas on the monolayer at the air-water interface. We believe that the compositions of the mixtures were conserved up to the first collapse pressure so that no squeeze out was seen from the monolayer at the air-water interface. There is a significant decrease in the mean molecular area for the mixtures at the second collapse pressure of $41 \mathrm{mN} / \mathrm{m}$. The decrease in the mean molecular area would not only be due to the conformational changes of the molecules, because they are already compressed to their minimal area at the end of the first collapse plateau, but also to the squeeze out of the $\mathrm{PEG}_{40} \mathrm{St}$ molecules from the monolayer to the subphase at the air-water interface causing the mean molecular area to decrease.

The DSPC and PEG ${ }_{40}$ St molecules were preserved in the monolayer at pressures less than the first collapse pressure. At the surface pressure of $33 \mathrm{mN} / \mathrm{m}$, the mean molecular areas for the mixtures were shown to increase slightly with the emulsifier content as shown in Fig. 1d. When the amounts of the two components were conserved, the measured surface area must be the area occupied by both components $\left(A_{12}=x_{1} .<A_{1}>+x_{2} \cdot<A_{2}>\right)$. Because the change in the mean molecular area for DSPC, $\left\langle A_{1}\right\rangle$, is relatively too small at around the collapse pressure, the DSPC molecules can be considered as rigid so that they would be a "reporting molecule" for the $\mathrm{PEG}_{40} \mathrm{St}$ molecules in the condensed monolayers. Therefore, the mean molecular area for DSPC, $\left.<A_{1}\right\rangle$, would be approximately equal to its ideal mean molecular area, $A_{1}$, obtained from its pure isotherm at the specified surface pressure. At the first collapse pressure where the DSPC and $\mathrm{PEG}_{40} \mathrm{St}$ molecules were relatively compressed, the average molecular area for the $\mathrm{PEG}_{40} \mathrm{St}$ molecules can be calculated before they undergo a collapse or squeeze out. As shown in Fig. 4, the mean molecular area for DSPC and $\mathrm{PEG}_{40} \mathrm{St}$ molecules were obtained from their pure isotherms at the first collapse pressure of $33 \mathrm{mN} / \mathrm{m}$. As shown in the figure, the mean molecular areas for DSPC were about $45 \AA^{2} /$ molecule whereas the mean molecular areas for $\mathrm{PEG}_{40}$ St were about $38 \AA^{2} /$ molecule and these mean molecular areas did not significantly changed for different emulsifier contents of the mixtures. The figure shows an increasing trend for the calculated mean molecular area for $\mathrm{PEG}_{40} \mathrm{St}$ in the mixture at diferrent emulsifier contents. The mean molecular area was calculated to be about $28 \AA^{2} /$ molecule for the $9: 1$ composion and $50 \AA^{2}$ / molecule for the 5:5 composition. The mean molecular area at the 9:1 composition is somewhat lower than the mean molecular area obtained 


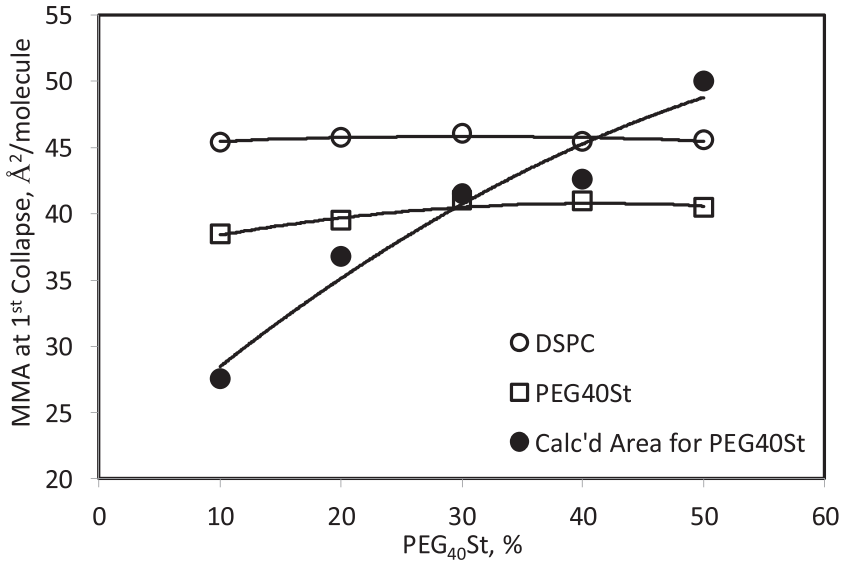

Fig. 4. The mean molecular areas measured for pure DSPC and pure $\mathrm{PEG}_{40} \mathrm{St}$ at the first collapse point. The surface areas for $\mathrm{PEG}_{40} \mathrm{St}$ in the DSPC/PEG $40 \mathrm{St}$ mixtures were calculated considering the mixture behave as an ideal mixture at the collapse point.

for the pure $\mathrm{PEG}_{40} \mathrm{St}$ molecule indicating that the $\mathrm{PEG}_{40} \mathrm{St}$ molecules were well dispersed within the DSPC molecules so that the DSPC molecules compressed the $\mathrm{PEG}_{40} \mathrm{St}$ molecules at molecular level and associated mostly with the stearate group of the $\mathrm{PEG}_{40} \mathrm{St}$ rather than PEG chains at the air-water interface. Therefore, the measured mean molecular area is close to the mean molecular area for the stearate group of the $\mathrm{PEG}_{40} \mathrm{St}$ molecules and a large portion of the PEG chains submerged within the subphase. The mean molecular area was measured to be about $50 \AA^{2} /$ molecule for the $5: 5$ composition indicating that the $\mathrm{PEG}_{40} \mathrm{St}$ molecules would be mostly phase separated and compressed over the PEG chains by the DSPC and therefore, higher average molecular areas were obtained for the $\mathrm{PEG}_{40}$ St molecules at higher emulsifier contents.

At surface pressures higher than the collapse pressure of any component, the mean molecular area for that component can be taken as the mean molecular area of its pure form at its collapse pressure. The mean molecular areas were measured to be about $40 \AA^{2} /$ molecule for DSPC at its collapse surface pressure of $59 \mathrm{mN} / \mathrm{m}$ and $30 \AA^{2} /$ molecule for $\mathrm{PEG}_{40} \mathrm{St}$ at its collapse surface pressure of $35 \mathrm{mN} / \mathrm{m}$.

The collapse pressures for the DSPC/ $\mathrm{PEG}_{40} \mathrm{St}$ mixtures at the last collapse point were obtained to be about $59 \mathrm{mN} / \mathrm{m}$ although the mean molecular area decreased almost linearly with the emulsifier content as shown in Fig. 5a. At the indicated collapse pressures, which are much higher than the collapse pressure of $35 \mathrm{mN} / \mathrm{m}$ for the $\mathrm{PEG}_{40} \mathrm{St}$, it was reported that all the emulsifier was squeeze-out from the 2D monolayer [6]. In this case, the mean molecular area for the DSPC in the monolayer can be estimated from the measured average molecular area for the mixtures omitting the mean molecular area for $\mathrm{PEG}_{40} \mathrm{St}$ $\left(A_{12}=x_{1} A_{1}+x_{2} A_{2}\right)$. Therefore, the measured mean molecular area for the mixture would be equal to the area occupied by the DSPC molecules with a mean molecular area of $40 \AA^{2}$ /molecule, which is reasonable because the variation in the mean molecular area is very small. Fig. $5 \mathrm{~b}$ shows the measured mean molecular area for the mixtures with respect to the calculated mean molecular area when the sole area occupied by DSPC and the $\mathrm{PEG}_{40}$ St component was omitted. As shown in the figure, the diagonal line represents the measured area would be equal to the calculated mean molecular area for the DSPC. However, the measured area for the mixtures is lower than the diagonal indicating that a small amount of DSPC would also be lost from the 2D monolayer at this late stage of the compression.

The squeeze out amount of $\mathrm{PEG}_{40} \mathrm{St}$ can be estimated from the measured surface area for the mixtures. The measured mean molecular area is the summation of the areas imposed by both DSPC and PEG $_{40} \mathrm{St}$ molecules and it was shown in Fig. 1d that the measured surface areas decreased with increasing the emulsifier content at each collapse
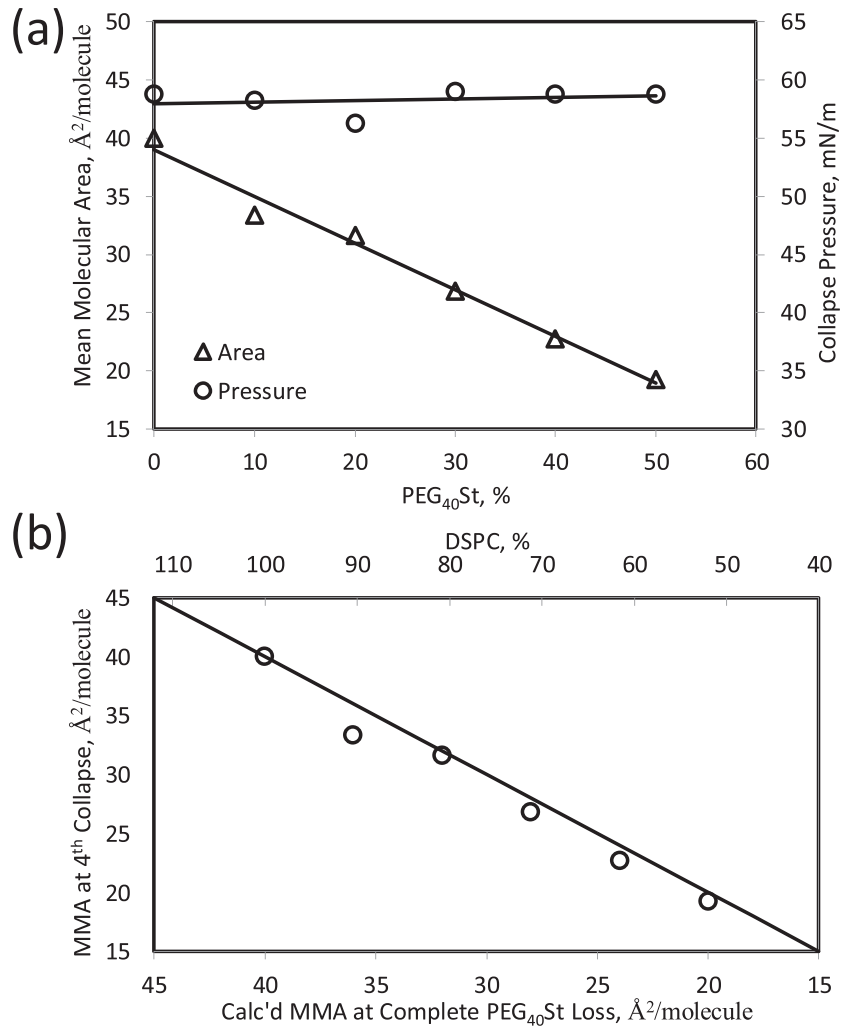

Fig. 5. (a) Collapse pressures and mean molecular areas for the DSPC/PEG ${ }_{40} \mathrm{St}$ mixtures at the last collapse point. (b) Measured mean molecular area for the mixture with respect to the calculated mean molecular area when $\mathrm{PEG}_{40} \mathrm{St}$ squeezed out fully from the $2 \mathrm{D}$ monolayer into the subphase.

points. The decrease in the measured surface area indicated that $\mathrm{PEG}_{40} \mathrm{St}$ molecules were lost at each collapse point and can be calculated using the equation $\left(A_{12}=x_{1} A_{1}+x_{2} A_{2} \cdot \boldsymbol{y}\right)$, where $\mathrm{y}$ is the percentage of $\mathrm{PEG}_{40} \mathrm{St}$ molecule contributed to the measured mean molecular area. The remaining of $y$ indicated the loss percentage of $\mathrm{PEG}_{40} \mathrm{St}$ from the monolayer. Here, DSPC is a rigid molecule and can be taken as the reporting molecule for the $\mathrm{PEG}_{40} \mathrm{St}$ because the change in the mean molecular area for DSPC was considered to be minimum or negligable, if any. Fig. 6a shows the estimated percentage of the $\mathrm{PEG}_{40} \mathrm{St}$ loss from the monolayer to the subphase at different collapse pressures. As shown in the figure, almost $93 \%$ of $\mathrm{PEG}_{40}$ St was lost at the end of the first collapse plateau for the 9:1 mixture and showed a decreasing trend for the higher $\mathrm{PEG}_{40} \mathrm{St}$ contents for the mixtures. For instance, almsost $82 \%$ and $53 \%$ of $\mathrm{PEG}_{40}$ St were lost for the 7:3 and 5:5 composions at the end of the first collapse plateau, respectively. Remaining of the $\mathrm{PEG}_{40} \mathrm{St}$ molecules were lost at the end of the second collapse plateau, where $20 \%$ of $\mathrm{PEG}_{40} \mathrm{St}$ were still present for the 5:5 mixture on the air-water interface. The $\mathrm{PEG}_{40} \mathrm{St}$ was entirely lost at the last collapse pressure for each mixtures studied.

The collapsed amounts of DSPC were estimated from the measured mean molecular area at the last collapse pressures. The fact that the measured surface area is lower than the estimated mean molecular area for the DSPC indicating that all the $\mathrm{PEG}_{40} \mathrm{St}$ and some of DSPC were lost from the 2D monolayer [6]. Then, the lost DSPC amount can be estimated from the measured mean molecular area at the last collapse pressure when the $\mathrm{PEG}_{40} \mathrm{St}$ molecules were omitted $\left(A_{12}=x_{1} A_{1} \cdot z+x_{2} A_{2}\right)$, where $\mathrm{z}$ is the percentage of DSPC contributed to the measured mean molecular area. The remaining of $\mathrm{z}$ indicated the percentage of DSPC loss from the monolayer. Fig. 6b shows the estimated percentage of the collapsed DSPC from the monolayer with different emulsifier contents. The DSPC molecules would also be lost from the monolayer into the subphase by associating with the $\mathrm{PEG}_{40} \mathrm{St}$ 

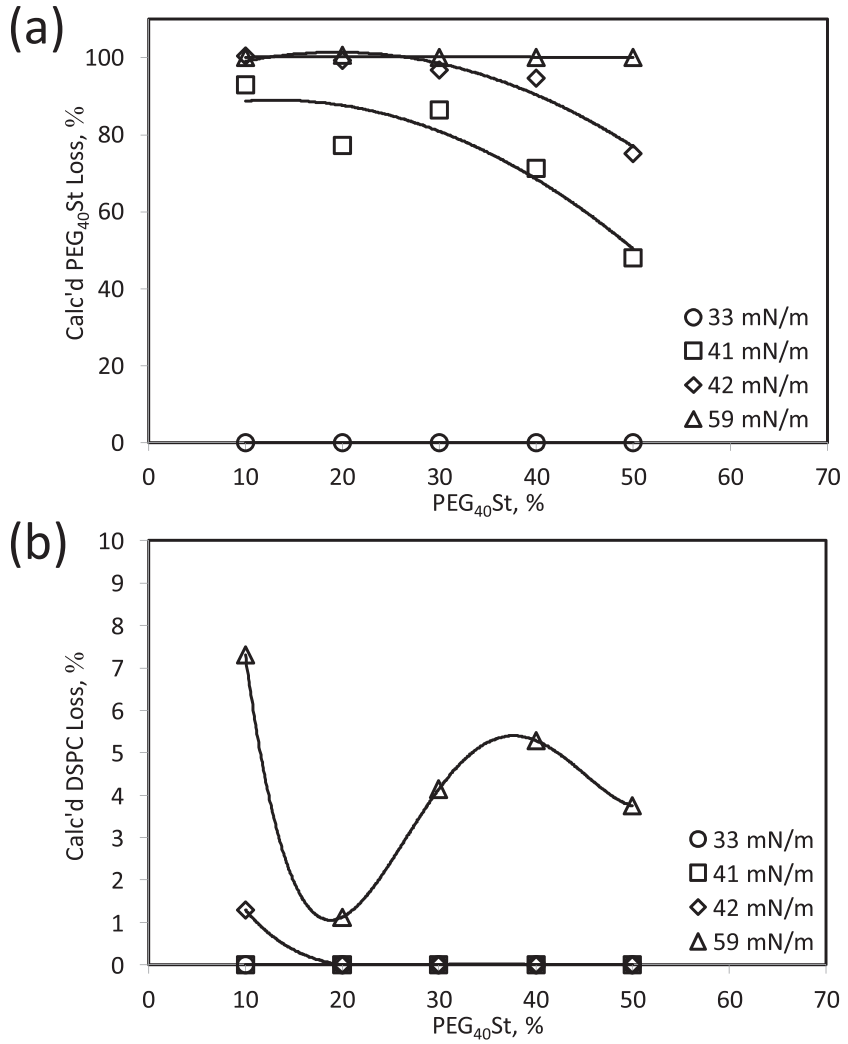

Fig. 6. (a) The calculated percent amount of $\mathrm{PEG}_{40} \mathrm{St}$ that squeeze out from the 2D monolayer at each DSPC/PEG ${ }_{40} \mathrm{St}$ mixtures at each collapse points. (b) The calculated percent amount of DSPC that moved from the 2D monolayer of the mixtures at each collapse point.

molecules. As shown in the figure, about $5 \%$ of DSPC molecules were lost for the mixtures, which can be considered relatively negligible.

\section{Conclusions}

A quantification method was developed to estimate the squeeze out amount of $\mathrm{PEG}_{40} \mathrm{St}$ from Langmuir isotherms of the DSPC/PEG ${ }_{40} \mathrm{St}$ monolayers at different molar ratios. The discontinuities on the Langmuir isotherm were always more extended for the first collapse plateaus and relatively shorter for the second collapse plateaus. The extent of the plateaus increased in proportional to the $\mathrm{PEG}_{40} \mathrm{St}$ content of the mixtures for the two collapse regions. The isotherms were analyzed considering that the DSPC molecules are relatively rigid at room temperature and their mean molecular area did not vary with relatively small deviations in the surface pressure, therefore, DSPC molecules were considered "reporter molecules" for the $\mathrm{PEG}_{40} \mathrm{St}$. It was also considered that the $\mathrm{PEG}_{40} \mathrm{St}$ molecules already compressed to their collapse area above its collapse pressures and its area can be taken as its mean molecular area at its collapse pressure at the end of the first and second collapse plateaus. Reproducible results were obtained for the mean molecular area of $40 \AA^{2} /$ molecule and $30 \AA^{2} /$ molecule for DSPC and $\mathrm{PEG}_{40} \mathrm{St}$ at their collapse pressures of $59 \mathrm{mN} / \mathrm{m}$ and $35 \mathrm{mN} / \mathrm{m}$, respectively. It was shown that $\mathrm{PEG}_{40} \mathrm{St}$ molecules compressed to different degree of molecular orientations depending on its content in the DSPC/ $\mathrm{PEG}_{40}$ St mixtures at pressures below the first collapse pressure. For instance, the $\mathrm{PEG}_{40} \mathrm{St}$ mean molecular areas were calculated to be $28 \AA^{2} /$ molecule for 9:1 composition and $50 \AA^{2} /$ molecule for $5: 5$ composition with an increasing trend with the $\mathrm{PEG}_{40} \mathrm{St}$ content at the collapse pressure of $33 \mathrm{mN} / \mathrm{m}$. As a result, at the end of the first collapse region, $93 \%, 82 \%$, and $53 \%$ of $\mathrm{PEG}_{40}$ St were lost for the 9:1, 7:3, and 5:5 mixtures, respectively, and showed a decreasing non-linear trend. Remaining $\mathrm{PEG}_{40} \mathrm{St}$ molecules were lost at the end of the second collapse plateau except for 5:5 mixture. At the end of the second collapse plateau, a $20 \%$ of $\mathrm{PEG}_{40}$ St were found to be still retained with the 5:5 mixture at the air-water interface and it squeezed out from the monolayer at the last collapse pressure for the DSPC. Retaining the $\mathrm{PEG}_{40} \mathrm{St}$ within the monolayer upon squeeze out is advantageous for the design of stable microbubble shell. Because the DSPC costs about \$169/ g-DSPC [53] and $\mathrm{PEG}_{40} \mathrm{St}$ costs about $\$ 0.22 / \mathrm{g}-\mathrm{PEG}_{40} \mathrm{St}$ [54], the cost for the $5: 5$ shell composition of DSPC/PEG 40 St mixture would be much cheaper compared to the customized composition of 9:1 molar ratio. It was concluded that increasing PEG $_{40}$ St content could not only increase the microbubble stability but also it decreases the product cost for the ultrasound contrast agents.

\section{Acknowledgements}

The Scientific and Technological Research Council of Turkey (TUBITAK) is gratefully acknowledged for the financial support provided under Project No. of 113M270. The author also thanks Elif Seniz Bolukcu for help conducting part of the experiments.

\section{References}

[1] E.G. Schutt, D.H. Klein, R.M. Mattrey, J.G. Riess, Injectable microbubbles as contrast agents for diagnostic ultrasound imaging: the key role of perfluorochemicals, Angew. Chem. Int. Ed. 42 (2003) 3218-3235.

[2] S. Hernot, A.L. Klibanov, Microbubbles in ultrasound-triggered drug and gene delivery, Adv. Drug. Deliv. Rev. 60 (2008) 1153-1166.

[3] S.R. Sirsi, M.A. Borden, Advances in ultrasound mediated gene therapy using microbubble contrast agents, Theranostics 2 (2012) 1208-1222.

[4] E.C. Unger, T. Porter, W. Culp, R. Labell, T. Matsunaga, R. Zutshi, Therapeutic applications of lipid-coated microbubbles, Adv. Drug. Deliv. Rev. 56 (2004) 1291-1314.

[5] R.H. Abou-Saleh, M. Swain, S.D. Evans, N.H. Thomson, Poly(ethylene glycol) lipidshelled microbubbles: abundance, stability, and mechanical properties, Langmuir 30 (2014) 5557-5563.

[6] M.A. Borden, G. Pu, G.J. Runner, M.L. Longo, Surface phase behavior and microstructure of lipid/PEG-emulsifier monolayer-coated microbubbles, Colloid Surf. B 35 (2004) 209-223.

[7] J.N. Israelachvili, D.J. Mitchell, B.W. Ninham, Theory of self-assembly of hydrocarbon amphiphiles into micelles and bilayers, J. Chem. Soc. Faraday Trans. 2 (72) (1976) 1525-1568.

[8] M.M. Kozlov, D. Lichtenberg, D. Andelman, Shape of phospholipid/surfactant mixed micelles: cylinders or disks? Theoretical analysis, J. Phys. Chem. B 101 (1997) 6600-6606.

[9] A.K. Kenworthy, S.A. Simon, T.J. Mcintosh, Structure and phase-behavior of lipid suspensions containing phospholipids with covalently attached poly(ethylene glycol), Biophys. J. 68 (1995) 1903-1920.

[10] K. Edwards, M. Johnsson, G. Karlsson, M. Silvander, Effect of polyethyleneglycolphospholipids on aggregate structure in preparations of small unilamellar liposomes, Biophys. J. 73 (1997) 258-266.

[11] M.A. Borden, M.L. Longo, Dissolution behavior of lipid monolayer-coated, air-filled microbubbles: effect of lipid hydrophobic chain length, Langmuir 18 (2002) 9225-9233.

[12] G. Pu, M.A. Borden, M.L. Longo, Collapse and shedding transitions in binary lipid monolayers coating microbubbles, Langmuir 22 (2006) 2993-2999.

[13] J.J. Kwan, M.A. Borden, Microbubble dissolution in a multigas environment, Langmuir 26 (2010) 6542-6548.

[14] G. Pu, M.L. Longo, M.A. Borden, Effect of microstructure on molecular oxygen permeation through condensed phospholipid monolayers, J. Am. Chem. Soc. 127 (2005) 6524-6525.

[15] J.A. Feshitan, C.C. Chen, J.J. Kwan, M.A. Borden, Microbubble size isolation by differential centrifugation, J. Colloid Interface Sci. 329 (2009) 316-324.

[16] E.J. Swanson, V. Mohan, J. Kheir, M.A. Borden, Phospholipid-stabilized microbubble foam for injectable oxygen delivery, Langmuir 26 (2010) 15726-15729.

[17] D.J. Cox, J.L. Thomas, Ultrasound-induced dissolution of lipid-coated and uncoated gas bubbles, Langmuir 26 (2010) 14774-14781.

[18] C. Allen, N. Dos Santos, R. Gallagher, G.N.C. Chiu, Y. Shu, W.M. Li, S.A. Johnstone, A.S. Janoff, L.D. Mayer, M.S. Webb, M.B. Bally, Controlling the physical behavior and biological performance of liposome formulations through use of surface grafted poly(ethylene glycol), Biosci. Rep. 22 (2002) 225-250.

[19] M.L. Immordino, F. Dosio, L. Cattel, Stealth liposomes: review of the basic science, rationale, and clinical applications, existing and potential, Int. J. Nanomed. 1 (2006) 297-315.

[20] H. Mulvana, E. Stride, J.V. Hajnal, R.J. Eckersley, Temperature dependent behavior of ultrasound contrast agents, Ultrasound Med. Biol. 36 (2010) 925-934.

[21] S. Garg, A.A. Thomas, M.A. Borden, The effect of lipid monolayer in-plane rigidity on in vivo microbubble circulation persistence, Biomaterials 34 (2013) 6862-6870.

[22] C.C. Chen, M.A. Borden, The role of poly(ethylene glycol) brush architecture in complement activation on targeted microbubble surfaces, Biomaterials 32 (2011) 
6579-6587.

[23] M.A. Borden, M.L. Longo, The dependence of lipid-coated microbubble dissolution behavior on acyl chain length, Biophys. J. 82 (2002) 35a-35a.

[24] M.A. Borden, G. Pu, M.L. Longo, P.A. Dayton, K.W. Ferrara, Phase behavior and transport properties of the lipid-monolayer shell of a microbubble, Abstr. Pap. Am. Chem. Soc. 230 (2005) U1072-U1073.

[25] J.J. Kwan, M.A. Borden, Lipid monolayer collapse and microbubble stability, Adv. Colloid Interface 183 (2012) 82-99.

[26] W. Wang, C.C. Moser, M.A. Wheatley, Langmuir trough study of surfactant mixtures used in the production of a new ultrasound contrast agent consisting of stabilized microbubbles, J. Phys. Chem. 100 (1996) 13815-13821.

[27] Z.W. Xing, H.T. Ke, J.R. Wang, B. Zhao, X.L. Yue, Z.F. Dai, J.B. Liu, Novel ultrasound contrast agent based on microbubbles generated from surfactant mixtures of span 60 and polyoxyethylene 40 stearate, Acta Biomater. 6 (2010) 3542-3549.

[28] E. Aumaitre, D. Vella, P. Cicuta, On the measurement of the surface pressure in Langmuir films with finite shear elasticity, Soft Matter 7 (2011) 2530-2537.

[29] M.A. Borden, Microbubble dispersions of natural lung surfactant, Curr. Opin. Colloid Interface Sci. 19 (2014) 480-489.

[30] T.H. Chou, I. Chu, Thermodynamic characteristics of DSPC/DSPE-PEG2000 mixed monolayers on the water subphase at different temperatures, Colloids Surf. B 27 (2003) 333-344.

[31] V. Tsoukanova, C. Salesse, Mixing behavior of a poly(ethylene glycol)-grafted phospholipid in monolayers at the air/water interface, Langmuir 24 (2008) 13019-13029.

[32] M.A. Borden, G.V. Martinez, J. Ricker, N. Tsvetkova, M. Longo, R.J. Gillies, P.A. Dayton, K.W. Ferrara, Lateral phase separation in lipid-coated microbubbles, Langmuir 22 (2006) 4291-4297.

[33] C. Ybert, W.X. Lu, G. Moller, C.M. Knobler, Collapse of a monolayer by three mechanisms, J. Phys. Chem. B 106 (2002) 2004-2008.

[34] K.Y.C. Lee, Collapse mechanisms of Langmuir monolayers, Annu. Rev. Phys. Chem. 59 (2008) 771-791.

[35] T.R. Baekmark, G. Elender, D.D. Lasic, E. Sackmann, Conformational transitions of mixed monolayers of phospholipids and polyethylene oxide lipopolymers and interaction forces with solid surfaces, Langmuir 11 (1995) 3975-3987.

[36] A. Lucero, M.R.R. Nino, A.P. Gunning, V.J. Morris, P.J. Wilde, J.M.R. Patino, Effect of hydrocarbon chain and $\mathrm{pH}$ on structural and topographical characteristics of phospholipid monolayers, J. Phys. Chem. B 112 (2008) 7651-7661.

[37] J. Majewski, T.L. Kuhl, M.C. Gerstenberg, J.N. Israelachvili, G.S. Smith, Structure of phospholipid monolayers containing poly(ethylene glycol) lipids at the air-water interface, J. Phys. Chem. B 101 (1997) 3122-3129.

[38] D. Takamoto, M. Lipp, A. Von Nahmen, K.Y.C. Lee, A. Waring, J. Zasadzinski, Interaction of lung surfactant proteins with anionic phospholipids, Biophys. J. 81 (2001) 153-169.

[39] M.A. Borden, G. Pu, G.J. Runner, M.L. Longo, Surface phase behavior and microstructure of lipid/PEG-emulsifier monolayer-coated microbubbles, Colloids Surf. B
35 (2004) 209-223

[40] C.M. Hollinshead, R.D. Harvey, D.J. Barlow, J.R.P. Webster, A.V. Hughes, A. Weston, M.J. Lawrence, Effects of surface pressure on the structure of distearoylphosphatidylcholine monolayers formed at the air/water interface, Langmuir 25 (2009) 4070-4077.

[41] P. Wydro, S. Knapczyk, M. Łapczyńska, Variations in the condensing effect of cholesterol on saturated versus unsaturated phosphatidylcholines at low and high sterol concentration, Langmuir 27 (2011) 5433-5444.

[42] I. Kubo, S. Adachi, H. Maeda, A. Seki, Phosphatidylcholine monolayers observed with Brewster angle microscopy and $\pi$-A isotherms, Thin Solid Films 393 (2001) $80-85$.

[43] K. Tanwir, V. Tsoukanova, Lateral distribution of a poly(ethylene glycol)-grafted phospholipid in phosphocholine monolayers studied by epifluorescence microscopy, Langmuir 24 (2008) 14078-14087.

[44] O. Tirosh, Y. Barenholz, J. Katzhendler, A. Priev, Hydration of polyethylene glycolgrafted liposomes, Biophys. J. 74 (1998) 1371-1379.

[45] Z. Xing, H. Ke, J. Wang, B. Zhao, X. Yue, Z. Dai, J. Liu, Novel ultrasound contrast agent based on microbubbles generated from surfactant mixtures of span 60 and polyoxyethylene 40 stearate, Acta Biomater. 6 (2010) 3542-3549.

[46] Y. Shen, R.L. Powell, M.L. Longo, Interfacial and stability study of microbubbles coated with a monostearin/monopalmitin-rich food emulsifier and PEG40 stearate, J. Colloid Interface Sci. 321 (2008) 186-194.

[47] F. Albertorio, A.J. Diaz, T.L. Yang, V.A. Chapa, S. Kataoka, E.T. Castellana, P.S. Cremer, Fluid and air-stable lipopolymer membranes for biosensor applications, Langmuir 21 (2005) 7476-7482.

[48] M.M. Lozano, M.L. Longo, Complex formation and other phase transformations mapped in saturated phosphatidylcholine/DSPE-PEG2000 monolayers, Soft Matter 5 (2009) 1822-1834.

[49] R.J. El-Khouri, S.L. Frey, A.W. Szmodis, E. Hall, K.J. Kauffman, T.E. Patten, K.Y.C. Lee, A.N. Parikh, A stripe-to-droplet transition driven by conformational transitions in a binary lipid-lipopolymer mixture at the air-water interface, Langmuir 27 (2011) 1900-1906.

[50] P. Wydro, K. Witkowska, The interactions between phosphatidylglycerol and phosphatidylethanolamines in model bacterial membranes: the effect of the acyl chain length and saturation, Colloids Surf. B 72 (2009) 32-39.

[51] K. Hac-Wydro, K. Jedrzejek, P. Dynarowicz-Latka, Effect of saturation degree on the interactions between fatty acids and phosphatidylcholines in binary and ternary Langmuir monolayers, Colloid Surf. B 72 (2009) 101-111.

[52] K.Y.C. Lee, A. Gopal, A. von Nahmen, J.A. Zasadzinski, J. Majewski, G.S. Smith, P.B. Howes, K. Kjaer, Influence of palmitic acid and hexadecanol on the phase transition temperature and molecular packing of dipalmitoylphosphatidyl-choline monolayers at the air-water interface, J. Chem. Phys. 116 (2002) 774-783.

[53] Avanti, March 2018.

[54] Sigma-Aldrich, March 2018. 